\title{
Numerical Analysis of a Trapezoidal Microchannel for Hydrodynamic Detachment of Cells
}

\author{
Jessanne Y. Lichtenberg, Yue Ling, Seunghyun Kim
}

\begin{abstract}
Hydrodynamic shear force along the bottom microchannel wall has been utilized in cell adhesion studies to detach cells in microfluidic channels. Due to the small dimensions of microfluidic channels, the shear stress produced in a conventional microchannel is dependent mainly on the fluid velocity and channel height. The wall shear force magnitude increases as the channel height is reduced. However, a reduced channel height decreases the sample volume to be contained in the fluidic channel and also increases the pressure drop significantly which may fail the fluidic device. In this study, a novel microchannel with a trapezoidal structure was investigated using computational fluid dynamics simulations. The key fluidic properties, including wall shear stress, sample volume, and pressure drop of the trapezoidal microchannel are compared with those of a conventional straight channel with a reduced channel height. We found the trapezoidal structure produces a wall shear stress of $5 \mathrm{~Pa}$ in the region of interest similar to that of the straight channel with a small channel height $(50 \mu \mathrm{m})$ while having less than 30 percent pressure drop. Additionally, the pressure drop can be reduced by modifying the geometry of the trapezoidal channel to minimize pressure loss.
\end{abstract}

Keywords: computational fluid dynamics, microchannel, microfluidics.

\section{INTRODUCTION}

Cell adhesion has been investigated for many cell biology and biomedical applications, such as studying cell signaling pathways, tissue engineering, biomaterials for implantable sensors, cancer metastasis, and the adhesion properties of normal and cancerous cells [1]. Cell adhesion studies can be classified into attachment or detachment events, both of which can be further categorized into population or single cell approaches. Methods to study cell population attachment include wash assays, resonance frequency, and microfluidics. Techniques to study the detachment of cell populations include centrifugation, spinning disk, flow chamber, or microfluidics. Methods to study single cell attachment include micropatterning, 3D hydrogels, and polyacrylamide-traction force microscopy (PA-TFM). Single cell detachment studies either focus on whole cells or single bonds. Whole cell detachment methods include cytodetachment, micropipette aspiration, or microfluidics.

Revised Manuscript Received on April 18, 2020.

* Correspondence Author

Jessanne Y. Lichtenberg, Department of Electrical and Computer Engineering, Baylor University, Waco, Texas, USA. Email: jess_lichtenberg1@baylor.edu

Yue Ling, Department of Mechanical Engineering, Baylor University, Waco, Texas, USA. Email: Stanley_ling@baylor.edu

Seunghuyn Kim*, Department of Electrical and Computer Engineering, Baylor University, Waco, Texas, USA. Email: seunghyun_kim@baylor.edu

(c) The Authors. Published by Blue Eyes Intelligence Engineering and Sciences Publication (BEIESP). This is an open access article under the CC BY-NC-ND license (http://creativecommons.org/licenses/by-nc-nd/4.0/)
Single bond detachment events are studied using single cell force spectroscopy (SCFS), such as atomic force microscopy (AFM) probe, biomembrane probe, or optical tweezer. Of the whole cell detachment methods, cytodetachment, which uses an AFM probe, and optical tweezer methods require specialized equipment and can damage the cell. Alternatively, microfluidics is straightforward, non-invasive, and allows for real-time measurement. Therefore, the microfluidics can be an effective tool for both population or single cell-based cell adhesion studies [1]. For the effective microfluidic detachment of cells, a sufficient wall shear stress must be produced in order to remove a cell from a microchannel surface. However, the flow rate in microfluidic channels is generally low due to the low Reynolds number [2], where $R e=\frac{\rho \overline{U h}}{\eta}$ is a measure of the relative importance of fluid inertia compared to viscous forces $(h, \bar{U}, \rho, \eta$ are the channel height, the mean fluid velocity, the fluid density, and fluid viscosity, respectively). In a conventional microfluidic channel the only way to achieve large shear stress while preserving the low volumetric flow rate is to reduce the channel height $[3,4]$. However, as the microchannel height is reduced, the pressure drop across the channel input and output will be significantly increased and the total volume contained in the fluidic device will be limited. We propose a novel trapezoidal microchannel to resolve this challenge. The main benefit of the new design is the lowered pressure drop across the channel. Other benefits include increasing the sample volume, while maintaining high shear stress at the region of interest (ROI) where the cell detachment occurs. The trapezoidal channel exhibits a reduced height only at the ROI; as a result, a higher shear stress is achieved with other benefits of larger channel height retained. The larger channel volume on the two sides of the trapezoidal structure allow for a greater sample volume to be held, while conserving a modest pressure drop. We, first, investigated conventional straight channels to determine the channel height that produces a critical shear stress on the surface to detach a model cell based on a reference analytically and numerically using a computational fluid dynamic (CFD) solver, Gerris [5]. Then, the CFD simulation was used to optimize the trapezoidal structure to minimize the pressure loss at the sharp corners and finally determine the shear stress at ROI, pressure drop, and fluid volume of a trapezoidal structure compared to a straight channel.

\section{METHODOLOGY}

Many studies have focused on theoretical models and experimental demonstration for cell detachment under a shearing flow [6-9].

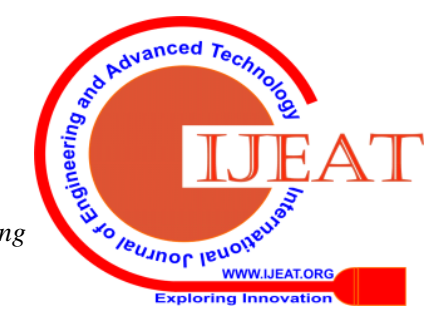


Gaver and Kute demonstrated the fluid shear stress on a cell is equal to the shear stress at the wall for $R / h<0.25$ where $R$ is the cell diameter and $h$ is the height of the microchannel [7].

Couzon et al. furthered this work to develop an equation for critical wall shear stress $\left(\tau_{C}\right)$ or the shear stress needed to remove an adhered cell to a channel wall,

$$
\tau_{\mathrm{C}}=\mathrm{Nf}_{\mathrm{ad}} \frac{1}{4 \mathrm{R}^{2}} \frac{\left(1-\left(\frac{\mathrm{R}}{\mathrm{h}}\right)^{2}\right)^{\frac{5}{2}}}{3.19+0.65 \frac{\mathrm{R}}{\mathrm{h}}+4.34\left(\frac{\mathrm{R}}{\mathrm{h}}\right)^{2}}
$$

where $f_{a d}$ is the force of each focal adhesion site on the cell (binding mechanism of cell to substrate) in the direction of flow, and $N$ is the number of focal adhesion sites [6]. For small channel height $(h<100 \mu \mathrm{m}), \tau_{C}$ increases rapidly with $h$. The $\tau_{C}$ for channels with larger height increases much slower and plateaus when $h$ becomes large. Equation (1) was used to estimate the average force per focal adhesion site of cancer cells adhered to a microchannels of varying heights . They used a T24 cell with a cell diameter, $R$, of $15 \mu \mathrm{m}$, channel heights from 60 to $260 \mu \mathrm{m}$, a width of $1 \mathrm{~mm}$, to estimate a total binding force, $N f_{a d}$, of $12 \mathrm{nN}$, and $\tau_{C}=5$ $\mathrm{Pa}$. This result is similar to available or estimated values of cell adhesion for different cell types [6].

We used a simplified version of (1) to perform analytical calculations to the critical wall shear that would be produced for a given flow rate and channel height. We also calculated the pressure gradient that would result from the same parameters. Then we calculated the channel height needed to detach a cell with a certain diameter and total binding force. From these analytical calculations, we identified key limitations in the geometry of traditional straight microfluidic channels.

To overcome the limitations of the straight microchannel, we designed a novel microfluidic design that would enable a high shear stress and low pressure drop. The unique geometry does not allow for the analytical calculations to solve for wall shear stress, so we relied on CFD simulations. The simulations were performed using the solver, Gerris, on the Baylor University supercomputing cluster, Kodiak. The computational domain is a rectangle. The length of the domain is 20 times of the height. The geometry of the bottom wall of the channel was specified as immersed solid in the computational domain. No-slip boundary condition is invoked on the immersed solid surface. The details of the channel geometry are given later. The fluid density was defined as $997 \mathrm{~kg} / \mathrm{m}^{3}$ and fluid viscosity of $0.89 \mathrm{mPa} \cdot \mathrm{s}$. The inlet fluid velocity was calculated from the calculated flow rate, discussed later. The computational domain was discretized by a quad-tree mesh and the mesh was refined with a maximum level of 8 and minimum level of 3, based on the estimated discretization error for the fluid velocity. The top boundary of the channel was defined as a no-slip wall. The numerical results were visualized with the software VisIt.

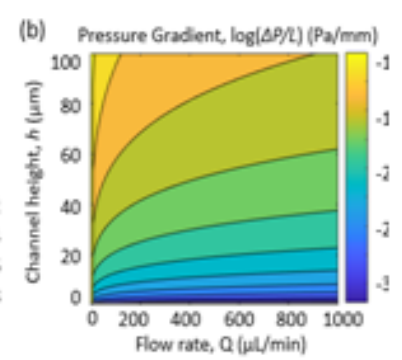

Fig, 1, Contour plot of (a) log scale wall shear stress $(\mathrm{Pa})$ and $(\mathrm{b}) \log$ scale pressure gradient $(\mathrm{Pa} / \mathrm{m})$ produced by different channel heights over a range of flow rates. The aspect ratio of the channel is fixed where $w$ is 10 times larger than $h$.

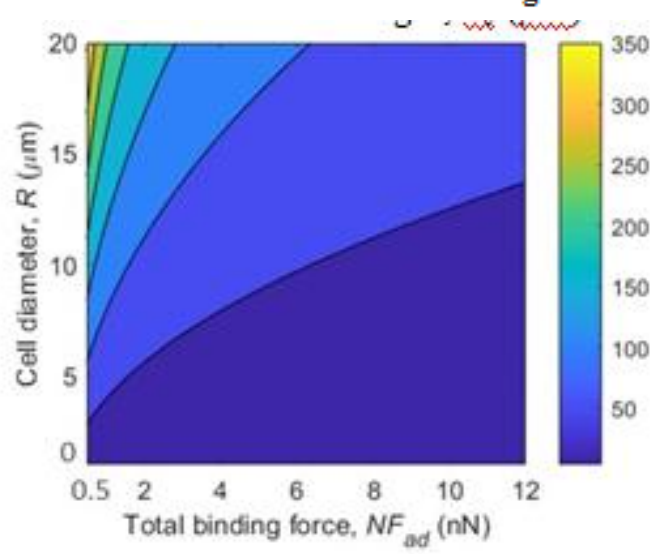

Fig, 2. Contour plot of the critical channel height needed for a given cell diameter and adhesion force, based on experimental data in Couzon et al. A constant $Q / w=2.34 \times 10^{-6} \mathrm{~m}^{2} / \mathrm{s}$ and $N=40$ are used.

\section{RESULTS}

\section{A. Straight Microchannel}

Table L. Microfluidic fluid dynamics results for tro analy̦tical cases (Aand B), and four simulated cases $(\mathrm{C}$ to $\mathrm{F}$, where $\mathrm{A}$ to $\mathrm{D}$ are straight microchannels and $\mathrm{E}$ to $\mathrm{F}$ are trapezoidal microchannels. Aconstant $Q / w=2,34 \times 10^{-6} \mathrm{~m}^{2} / \mathrm{s}$ and $L=1 \mathrm{~cm}$ is used.

\begin{tabular}{|c|c|c|c|c|c|c|c|}
\hline Vaniable & \multicolumn{2}{|c|}{ Analytical } & \multicolumn{4}{|c|}{ Simulated } & Units \\
\hline Channel & A & B & C & D & E & $\mathrm{F}$ & \\
\hline h & 50 & 500 & 50 & 500 & 500 & 500 & $\mu \mathrm{m}$ \\
\hline$h_{g}$ & . & . & . & . & 50 & 50 & $\mu \mathrm{m}$ \\
\hline$L_{I}$ & . & - & - & - & 0.5 & 2.5 & $\mathrm{~mm}$ \\
\hline$U$ & 46.8 & 4.68 & 46.8 & 4.68 & 4.68 & 4.68 & $\mathrm{~mm} / \mathrm{s}$ \\
\hline sive & 5 & 0.05 & 4.98 & 0.049 & 4.96 & 4.96 & $\mathrm{~Pa}$ \\
\hline$\Delta P$ & 2000 & 2 & 1999 & 1.98 & 259 & 589 & $\mathrm{~Pa}$ \\
\hline$V$ & 0.25 & 25 & 0.25 & 25 & 21.8 & 19.8 & $\mu \mathrm{L}$ \\
\hline
\end{tabular}

Fig. 1a shows a map of the log of wall shear stress $\left(\tau_{w}\right)$ in a microchannel with different flow rates and channel heights. The fluid viscosity of water was used ( $\eta=0.89 \mathrm{mPa} \cdot \mathrm{s})$, which is similar to the viscosity of phosphate buffered saline (PBS), a commonly used buffer solution in microfluidic applications.

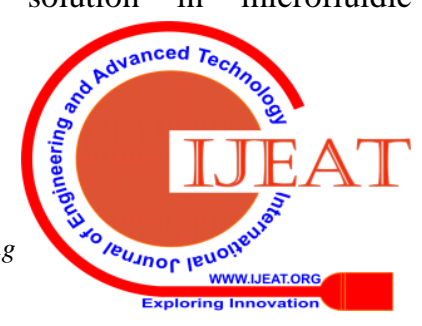


For the ranges shown, there is the inverse relationship between channel height and wall shear stress. It is clear that changing the channel height affects the wall shear stress more than changing the flow rate. The pressure drop, $\Delta P$, in a microchannel is directly proportional to the flow rate, $Q$, and channel length, $L$. Channel geometry such as convergence, divergence and corners can affect the pressure drop. Pressure is an important consideration in microfluidics because high pressure build-up can lead to the deformation or delamination of the microchannels, which are commonly fabricated using soft lithography or photolithography [10]. The equation $\frac{\Delta \mathrm{P}}{\mathrm{L}}=-\frac{12 \eta \mathrm{Q}}{\mathrm{wh}^{3}}$ was used to create a map of the log of pressure gradient in microchannel with different flow rates and channel heights (Fig. 1b). Note that we used a 1:10 channel height to channel width ratio, based on the standard geometry for a rectangular microchannel where $w>>h$ [11]. In order to produce a wall shear stress above $1 \mathrm{~Pa}$, the microchannel height must be less than $40 \mu \mathrm{m}$ for all the flow rates (Fig. 1a). However, the pressure gradient for channel heights less than $40 \mu \mathrm{m}$ is $2-3$ times greater than that with larger channel heights.

Based on a simplified version of (1) for $h<100 \mu \mathrm{m}$ [6], we derived an equation for critical channel height $\left(h_{c}\right)$, the channel height necessary to detach a cell with certain diameter and total binding force (2),

$$
\mathrm{h}_{\mathrm{C}}=\sqrt{\frac{76.56 \mathrm{R}^{2} \eta \mathrm{Q}}{\mathrm{Nf}_{\mathrm{ad}} \mathrm{W}}}
$$

where $Q$ is the volumetric flow rate, and $w$ is the microchannel width. Fig. 2 shows the critical channel height based on $R$ ranging from 1 to $20 \mu \mathrm{m}$ and $N f_{a d}$ ranging from
1 to $12 \mathrm{nN}$, where a constant $Q / w=2.34 \times 10^{-6} \mathrm{~m}^{2} / \mathrm{s}$, and $N=$ 40 was used. As an example, for a 2D flow rate of $Q / w=2.34$ $\mathrm{x} 10^{-6} \mathrm{~m}^{2} / \mathrm{s}$, the critical channel height $\left(h_{c}\right)$ is $50 \mu \mathrm{m}$ (Channel A) to achieve a $\tau_{w}=5 \mathrm{~Pa}$ that matches the $\tau_{c}=5 \mathrm{~Pa}$, the critical shear stress for a T24 cell $\left(N f_{a d}=12 n N\right)$. For this Channel A with the channel height of $50 \mu \mathrm{m}$, the calculated pressure drop ( $\left.\Delta P=P_{\text {inlet }}-P_{\text {outlet }}\right)$ for a channel length $(L)$ of $1 \mathrm{~cm}$ is 2,000 Pa based on $Q / w=2.34 \times 10^{-6} \mathrm{~m}^{2} / \mathrm{s}$ while fluid velocity $(U)$ is $46.8 \mathrm{~mm} / \mathrm{s}$, calculated from $U=Q / w h$. The sample volume capacity of microchannel was calculated, based on a 1:10 channel height to channel width ratio. In Channel A with a width of $500 \mu \mathrm{m}$ and $L=1 \mathrm{~cm}$, the total volume of the microchannel is only $0.25 \mu \mathrm{l}$. One way to increase the volume of Channel A is to increase the channel length. However, that could significantly magnify the pressure drop, which can be risky as previously discussed.

To compare the results with a larger channel, we chose a height of $500 \mu \mathrm{m}$ (Channel B) based on dimensions in literature for cell detachment studies [12,13]. We used $Q / w=$ $2.34 \times 10^{-6} \mathrm{~m}^{2} / \mathrm{s}, L=1 \mathrm{~cm}$, and 1:10 channel ratio as the baseline parameters to compare calculations and simulations throughout this paper. The $\tau_{w}$ produced by Channel B is 0.05 $\mathrm{Pa}$ which is obviously insufficient to detach cells from the surface. However, the $\Delta P$ for this channel is $2 \mathrm{~Pa}$ which is three orders of magnitude smaller than that for Channel A. The sample volume for channel B with a $w=5,000 \mu \mathrm{m}$ is 25 $\mu \mathrm{l}, 100$ times greater that of Channel A. The Reynolds number is also calculated to ensure laminar flow $(R e<2300)$. With a constant flow rate for both Channel $\mathrm{A}$ and $\mathrm{B}$, the Reynolds number is calculated using the fluid velocities in Table I to be 2.62 which is reasonable for

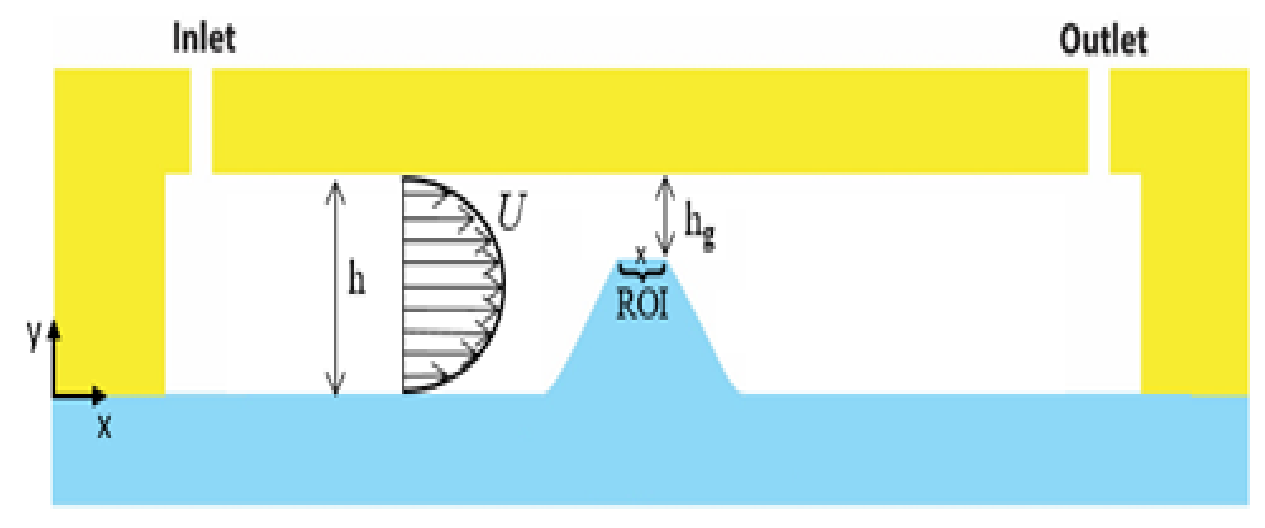

Fig, 3. Schematic of trapezoidal microchannel with the ROI in the center of the channel.
The height, h, and gap height, hg, are denoted. The " $\mathrm{x}$ " in the center of the channel
marks where the wall shear stress is calculated in this study.

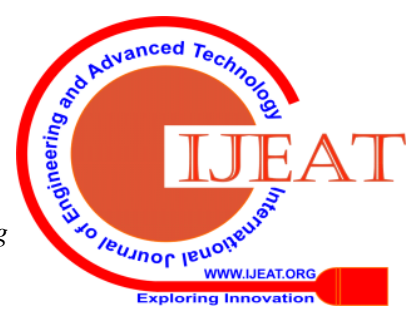




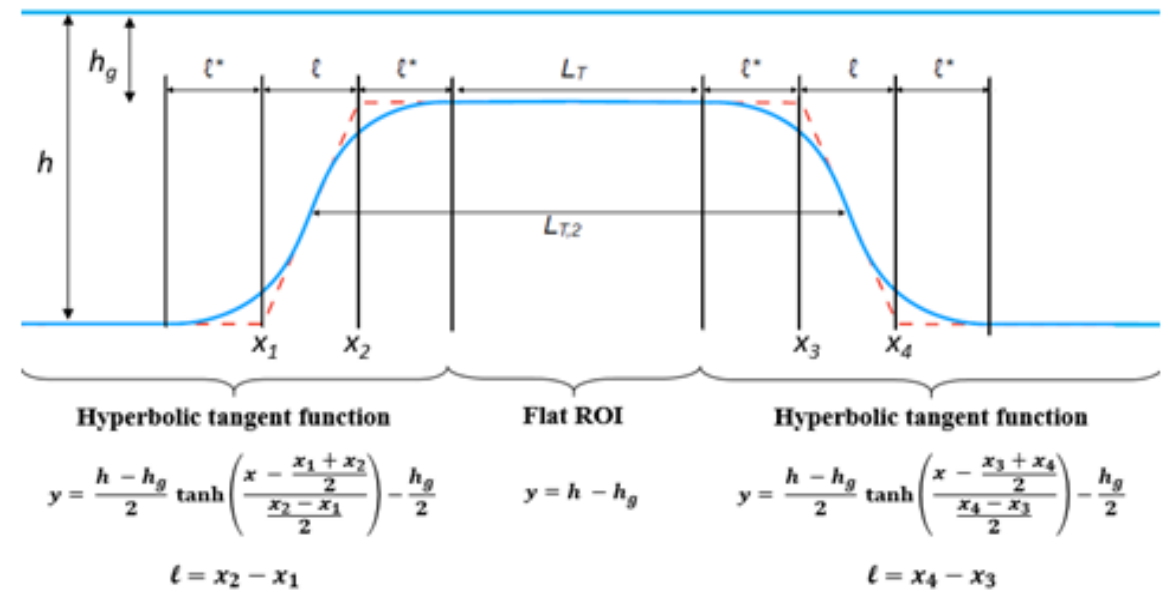

Fig, 4. Schematic of the trapezoidal channel geometry to optimize the design. The blue lines denote the channel wall, and key dimensions are labeled. The equations used to create the smooth channel surfaces are detailed for each part of the trapezoid.

microfluidics. The proposed trapezoidal microchannel takes advantages of both small and large height channel designs. With the trapezoidal microchannel, the critical shear stress is achieved at the region of interest (ROI), where cell detachment studies could be performed, while minimizing the overall channel pressure drop and increasing the sample volume capacity. The analytical calculation results for Channel A and B are summarized in Table I along with the CFD simulation results for straight channels and trapezoidal structures.

\section{B. Trapezoidal Microchannel}

Before we perform the CFD simulations for the trapezoidal structure, we validated the CFD simulation accuracy comparing to analytical calculation results using two straight channel cases. The simulated straight channels, Channel C and $\mathrm{D}$, are exactly same as Channel A and B, respectively. As shown in Table I, the calculated wall shear stress (C: $\tau_{w}=$ 4.98 Pa, D: $\left.\tau_{w}=0.049\right)$ and pressure drop (C: $\Delta P=1999 \mathrm{~Pa}$,

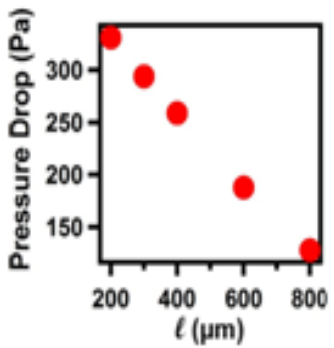

(a)

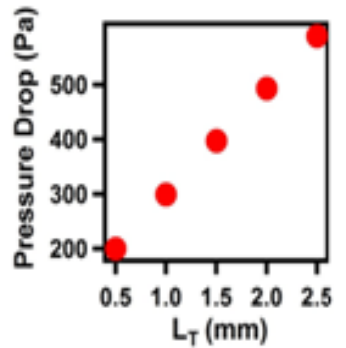

(b)
Fig, 5. The pressure drop as a function of (a) $\ell$ and (b) $L$. for $\ell=400 \mu \mathrm{m}$.

$\mathrm{D}: \Delta P=1.98 \mathrm{~Pa}$ ) agree well with the analytical solutions (A: $\tau_{w}=5 \mathrm{~Pa}$ and $\Delta P=2000 \mathrm{~Pa}, \mathrm{~B}: \tau_{w}=0.05 \mathrm{~Pa}$ and $\Delta P=2 \mathrm{~Pa}$ ).

The proposed trapezoidal microchannel design is shown in Fig. 3. It has a narrower It has a channel height at the ROI in the center of the channel (i.e., detachment area) to achieve a higher shear stress. A conventional trapezoidal channel with sloped sides and a flat top surface (red dashed line in Fig. 4) was first investigated. The calculated pressure drop for that channel was $372 \mathrm{~Pa}$. To test the pressure loss due to the sharp corners, a hyperbolic tangent function was used to optimize the trapezoidal structure with a smoother profile as shown in Fig. 4. The width of the hyperbolic tangent ( $l$ ) was varied, while the $\mathrm{L}_{\mathrm{T}, 2}$ (median length of trapezoidal structure) was kept constant at $2 \mathrm{~mm}$ and $\ell=\ell^{*}$. The results show the pressure drop decreases linearly with increasing $\ell$ (Fig. 5a). For $\ell=200 \mu \mathrm{m}$, the pressure drop was $331 \mathrm{~Pa}$ compared to a pressure drop of $125 \mathrm{~Pa}$ for $\ell=800 \mu \mathrm{m}$. We chose $\ell=400 \mu \mathrm{m}$ and simulated the trapezoidal channel structure with hyperbolic tangent sides to find the relationship between the pressure drop and $L_{T}$ (the top surface length of the trapezoidal structure). As shown in Fig. 5b, the results show the pressure drop increases proportionally to the $L_{T}$. An $L_{T}=0.5 \mathrm{~mm}$ results in a $\Delta P=200 \mathrm{~Pa}$ while an $L_{T}=2.5 \mathrm{~mm}$ produces a $\Delta P$ $=589 \mathrm{~Pa}$.

Channel E shown in Table I is a trapezoidal microchannel with a $L_{T}$ of $0.5 \mathrm{~mm}$ and its fluid velocity distribution calculated with CFD is shown in Fig. 6a. A close up view of the ROI at the top of the trapezoidal structure shows a uniform parabolic fluid velocity distribution. The fluid velocity profile for Channel $\mathrm{F}$ in Table I, at $2 \mathrm{~mm}$ and $\ell=\ell^{*}$. The results show the pressure drop decreases linearly with increasing $\ell$ (Fig. 5a). For $\ell=200 \mu \mathrm{m}$, the pressure drop was $331 \mathrm{~Pa}$ compared to a pressure drop of $125 \mathrm{~Pa}$ for $\ell=800 \mu \mathrm{m}$. We chose $\ell=400 \mu \mathrm{m}$ and simulated the trapezoidal channel structure with hyperbolic tangent sides to find the relationship between the pressure drop and $L_{T}$ (the top surface length of the trapezoidal structure). As shown in Fig. 5b, the which has a $L_{T}$ of $2.5 \mathrm{~mm}$, is almost same as that of Channel $\mathrm{E}$ except the longer top surface length. Fig. $6 \mathrm{~b}$ shows the velocity profiles for Channels $\mathrm{E}$ and $\mathrm{F}$ at $\mathrm{x}=4.75 \mathrm{~mm}$ compared to the straight channel (Channels A (analytical) and $\mathrm{C}$ ). The profiles are all similar to each other as expected, likely due to fully developed flow profile at the center of the $L_{T}$. The wall shear stress for the trapezoidal microchannels shown in Table I was calculated using the velocity profiles shown in Fig. 6b. As shown in Table I, Channel E and F produced similar wall shear stresses (4.96 $\mathrm{Pa})$.

Published By:

Blue Eyes Intelligence Engineering

\& Sciences Publication

(C) Copyriaht: All riahts reserved.

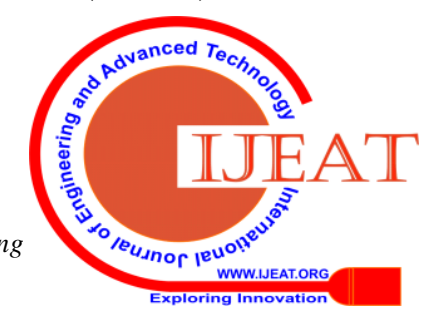


The pressure drop of trapezoidal channels are $259 \mathrm{~Pa}$ in Channel $\mathrm{E}$ and $589 \mathrm{~Pa}$ in Channel $\mathrm{F}$ due to longer shallow channel ROI. Comparing to the straight channel, the wall shear stress in Channel $\mathrm{E}$ is $99.5 \%$ of the wall shear stress in Channel C with $13 \%$ of the pressure drop. The wall shear stress in Channel F is the same as Channel E, with $29 \%$ of the pressure drop of Channel C. Additionally, the calculated fluid volumes (E: $21.8 \mu \mathrm{L}$ and $\mathrm{F}: 19.8 \mu \mathrm{L}$ ) are similar to Channels $\mathrm{B}$ and $\mathrm{D}$ with a larger channel height $(25 \mu \mathrm{L})$. The reduced channel height at the ROI does not limit the volume capability of the trapezoidal microchannels.

\section{CONCLUSION}

Analytical calculations showed a channel with a smaller height (Channel A: $50 \mu \mathrm{m}$ ) can produce a $\tau \mathrm{w}=5 \mathrm{~Pa}$ which is sufficient to detach a T24 cell for cell adhesion study while a channel with larger height (Channel B: 500 um) only produced a $\tau \mathrm{w}=0.05 \mathrm{~Pa}$ with the same flow rate. However, the pressure drop is much greater in Channel A (2000 Pa in A vs $2 \mathrm{~Pa}$ in $\mathrm{B})$ and the fluid volume is much less (A: $0.25 \mu \mathrm{L}$ vs. B: $25 \mu \mathrm{L})$.

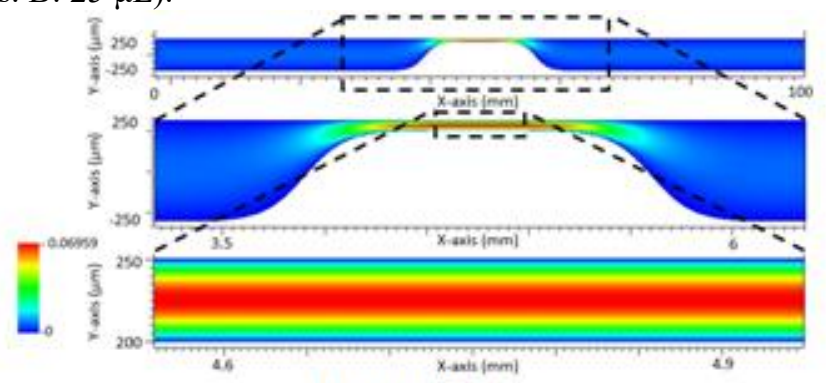

(a) Channel E

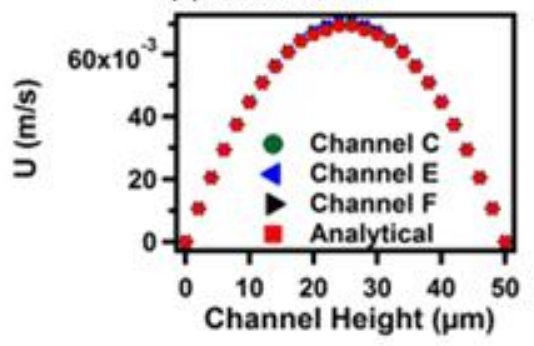

(b)

Fig. 6. (a) CFD simulation color plot of the velocity in Channel E, with a close up of the center of the channel where the shear stress was evaluated. (b) Plots of the velocity profiles for all 4 simulations compared to the analytical solution for a $50 \mu \mathrm{m}$ channel, at the center x-axis point of the channel. The velocity profile of the trapezoidal matches that of the straight channel cases.

As a solution to these limitations, we investigated a trapezoidal microchannel. CFD simulations demonstrated a similar wall shear stress was produced ( $\mathrm{E}$ and $\mathrm{F}$ : $4.96 \mathrm{~Pa}$ ) with much smaller pressure drop (E: $259 \mathrm{~Pa}$ and F: $589 \mathrm{~Pa}$ ) and larger fluid volumes (E: $21.8 \mu \mathrm{L}$ and $\mathrm{F}: 19.8 \mu \mathrm{L}$ ). The ability to generate a high critical wall shear stress with lowered pressure drop is a clear benefit to this design. Additionally, the isolation of the reduced height area to just the ROI allows for flexibility in the rest of the channel to incorporate other microfluidic components, and hold a larger fluid volume. These outcomes of this study demonstrate an approach to design a trapezoidal microchannel to achieve a critical wall shear stress for microfluidic applications such as cell detachment. Analytical equations can be used to determine the necessary gap height to achieve the desired wall shear stress. The ideal sample volume and pressure drop can be used to design the channel height of the microchannel other than ROI, and curvature of the hyperbolic functions of the trapezoidal microchannel.

\section{REFERENCES}

1. Ahmad Khalili, A.; Ahmad, M. R. A Review of Cell Adhesion Studies for Biomedical and Biological Applications. Int. J. Mol. Sci. 2015, 16 (8), 18149-18184

2. Huber, D.; Oskooei, A.; Casadevall i Solvas, X.; deMello, A.; Kaigala, G. V. Hydrodynamics in Cell Studies. Chem. Rev. 2018, 118 (4), 2042-2079.

3. Carré, A.; Mittal, K. L. Surface and Interfacial Aspects of Cell Adhesion; CRC Press, 2011.

4. Lu, H.; Koo, L. Y.; Wang, W. M.; Lauffenburger, D. A.; Griffith, L. G.; Jensen, K. F. Microfluidic Shear Devices for Quantitative Analysis of Cell Adhesion. Anal. Chem. 2004, 76 (18), 5257-5264.

5. "Gerris Flow Solver."

gfs.sourceforge.net/wiki/index.php/Main_Page

6. Couzon, C.; Duperray, A.; Verdier, C. Critical Stresses for Cancer Cell Detachment in Microchannels. Eur. Biophys. J. 2009, 38 (8), 1035-1047.

7. Gaver, D. P.; Kute, S. M. A Theoretical Model Study of the Influence of Fluid Stresses on a Cell Adhering to a Microchannel Wall. Biophys. J. 1998, 75 (2), 721-733.

8. Décavé, E.; Garrivier, D.; Bréchet, Y.; Bruckert, F.; Fourcade, B. Peeling Process in Living Cell Movement Under Shear Flow. Phys. Rev. Lett. 2002, 89 (10), 108-101.

9. Garrivier, D.; Décavé, E.; Bréchet, Y.; Bruckert, F.; Fourcade, B. Peeling Model for Cell Detachment. Eur. Phys. J. E 2002, 8 (1), 79-97.

10. W. Oh, K.; Lee, K.; Ahn, B.; P. Furlani, E. Design of Pressure-Driven Microfluidic Networks Using Electric Circuit Analogy. Lab. Chip 2012, 12 (3), 515-545.

11. Squires, T. M.; Quake, S. R. Microfluidics: Fluid physics at the nanoliter scale. Rev. Mod. Phys. 2005, 77 (3), 977-1026.

12. 12. Kou, S.; Pan, L.; van Noort, D.; Meng, G.; Wu, X.; Sun, H.; Xu, J.; Lee, I. A Multishear Microfluidic Device for Quantitative Analysis of Calcium Dynamics in Osteoblasts. Biochem. Biophys. Res. Commun. 2011, 408 (2), 350-355.

13. M. Walker, G.; C. Zeringue, H.; J. Beebe, D. Microenvironment Design Considerations for Cellular Scale Studies. Lab. Chip 2004, 4 (2), 91-97

\section{AUTHORS PROFILE}

Jessanne Y. Lichtenberg is a Ph.D. student in the Department of Biomedical Engineering at Virginia Commonwealth University. She earned a B.S. degree from LeTourneau University and M.S. degree from Baylor University. Her current research interests lie in using microfluidic devices to recapitulate the human body to study diseases such as breast cancer. She has been a member of the Society of Women Engineers since 2014, and was inducted into the Golden Key International Honour Society and Who's Who among Students in American Universities \& Colleges.

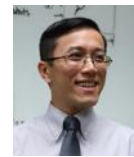

Dr. Yue Ling is currently an assistant professor in the Department of Mechanical Engineering at Baylor University. He obtained his $\mathrm{Ph} . \mathrm{D}$. degree in mechanical engineering at University of Florida in 2010. His research focuses on high-fidelity modeling and simulation of interfacia multiphase flows, including atomization, sprays, and droplet-based microfluidics. He has published 21 journal articles and 19 peer-reviewed conference papers.

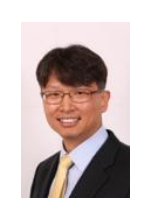

Dr. Seung Kim is an associate professor in the department of Electrical and Computer Engineering at Baylor University. He earned a Ph.D. degree in Optical Science and Engineering from University of Alabama in Huntsville in 2004. His expertise include optical biosensors, micro and nanofabrication processes, microfluidics, and integrated optics. He is a recipient of a NSF CAREER grant. He also received an outstand professor award at Baylor University in 2018 for outstanding research activities. He has 4 patents and 33 journal publications in addition to 55 conference publications.

Published By:

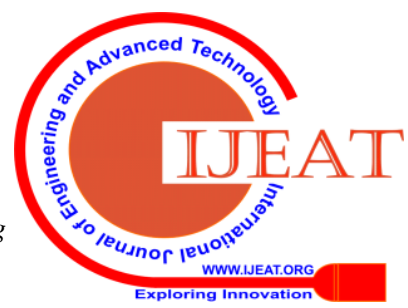

